\title{
Interference Cancellation for Hollow-Core Fiber Reference Cells
}

Seppä, Jeremias; Merimaa, Mikko; Merimaa, Mikko; Manninen, Albert; Triches, Marco; Hald, Jan; Lassila, Antti

Published in:

I E E E Transactions on Instrumentation and Measurement

Link to article, DOI:

10.1109/TIM.2015.2408800

Publication date:

2015

Link back to DTU Orbit

Citation (APA):

Seppä, J., Merimaa, M., Merimaa, M., Manninen, A., Triches, M., Hald, J., \& Lassila, A. (2015). Interference Cancellation for Hollow-Core Fiber Reference Cells. I E E E Transactions on Instrumentation and Measurement, 64(4). https://doi.org/10.1109/TIM.2015.2408800

\section{General rights}

Copyright and moral rights for the publications made accessible in the public portal are retained by the authors and/or other copyright owners and it is a condition of accessing publications that users recognise and abide by the legal requirements associated with these rights.

- Users may download and print one copy of any publication from the public portal for the purpose of private study or research.

- You may not further distribute the material or use it for any profit-making activity or commercial gain

- You may freely distribute the URL identifying the publication in the public portal

If you believe that this document breaches copyright please contact us providing details, and we will remove access to the work immediately and investigate your claim 


\title{
Interference Cancellation for Hollow-Core Fiber Reference Cells
}

\author{
Jeremias Seppä, Mikko Merimaa, Albert Manninen, Marco Triches, Jan Hald, and Antti Lassila
}

\begin{abstract}
Doppler-free saturated absorption spectroscopy of gases in hollow-core fiber (HCF)-based cells can be used for realizing new compact, robust, and portable frequency standards. In this paper, methods for cancelling interferences resulting from the optical connections between standard fiber and HCF and other factors such as varying coupling to HCF modes are investigated. Laser power modulation with simultaneous detection of ac and dc signal is used to separate saturated absorption from interferences. In addition, a technique of two piezoelectric stack actuators stretching the fiber at different locations is described. The presented experimental results demonstrate that $99 \%$ interference attenuation is readily attainable with the techniques. Frequency comb-referenced measurement of saturated acetylene absorption features near $1.54 \mu \mathrm{m}$, with fiber length and power modulation, is presented. For comparison, measurements and results of the same acetylene fiber, using a system having pump and probe beams at mutually shifted frequencies, are also given.
\end{abstract}

Index Terms-Frequency measurement standards, infrared spectra, interference cancellation, measurement techniques, metrology, optical fiber, spectroscopy.

\section{INTRODUCTION}

$\mathbf{S}$ ATURATED absorption spectroscopy usually requires beams of light passing through low-pressure gas in opposite directions along the same optical path, enabling observation of the center of the spectral transition without Doppler broadening. Using a hollow-core fiber (HCF)-based gas cell [1], [2] in an all-fiber arrangement improves robustness against optical misalignment, mechanical vibration, and temperature changes.

However, interference arising from interfaces between the optical components can be a severe problem. In traditional free-space optical arrangements for spectroscopy and laser stabilization, interference can be attenuated by dithering the end mirror [3]-[5] or by having the pump and probe light

Manuscript received August 22, 2014; revised January 21, 2015; accepted February 10, 2015. This work was supported by the European Metrology Research Programme (EMRP), JRP IND14 Frequency. The EMRP is supported by the participating countries within EURAMET and the European Union. The Associate Editor coordinating the review process was Dr. Lucas Di Lillo.

J. Seppä, M. Merimaa, A. Manninen, and A. Lassila are with the Centre for Metrology MIKES, VTT Technical Research Centre of Finland, Espoo FI-02151, Finland (e-mail: jeremias.seppä@mikes.fi; mikko.merimaa@mikes.fi; albert.manninen@mikes.fi; antti.lassila@mikes.fi).

M. Triches is with Danish Fundamental Metrology A/S, Kgs. Lyngby DK-2800, Denmark, and also with the Department of Fotonik Engineering, Technical University of Denmark, Kgs. Lyngby DK-2800, Denmark (e-mail: mt@dfm.dk).

J. Hald is with Danish Fundamental Metrology A/S, Kgs. Lyngby DK-2800, Denmark (e-mail: jha@dfm.dk).

Color versions of one or more of the figures in this paper are available online at http://ieeexplore.ieee.org.

Digital Object Identifier 10.1109/TIM.2015.2408800 beams crossing at a small angle instead of following the same optical path, causing also a shift to the measured spectrum. However, with the HCF cell it is not usually possible to reflect light back directly from the end of the cell nor to have the pump and probe beams at an angle. Introducing the light into the HCF at both ends by, e.g., having the HCF spliced between telecom fibers produces optical interfaces that can have high reflectivities and losses. Using, e.g., the end mirror and HCF-based arrangement results in complex interference effects from multiple optical interfaces and possibly also effects due to the mode structure of the fiber.

An alternative to using pump and probe light at the same wavelength is having the two light beams with a mutual frequency shift. This has been successfully used for cancelling majority of the interference effects by shifting the interference of the pump and probe light in the interfaces from dc to, e.g., megahertz range frequencies [1], [2]. Using pump and probe light at different wavelengths leads to a somewhat more complicated system, and in principle, attenuates a different subset of the possible interference effects.

In this paper two different methods to attenuate interference effects in HCF-based systems with an end mirror are investigated. First, a piezo modulation scheme, published in [6], using two piezos modulating both the $\mathrm{HCF}$ and the end mirror is shown to greatly reduce the interferences in a carbon dioxide-filled fiber spliced between standard single-mode fibers. Second, a method using laser power modulation and ac/dc detection is shown to be able to differentiate the nonlinear saturated absorption from the linear interference and linear absorption to a high degree in a low-pressure acetylene-filled fiber. The dc component of detector signal is detected in both setups using a digital voltmeter (DVM), and in the latter method the ac component is detected using a lock-in amplifier.

The acetylene-filled fiber component used in the latter experiment was made by Danish Fundamental Metrology (DFM), and has coupling lenses at both ends of the HCF. The laser frequency in the latter experiment is locked to atomic clock via frequency comb [7]. The comb repetition rate is stepped to scan the locked laser frequency.

As a third measurement method, recent results with the same HCF cell, using pump and probe light at different frequencies [8] are shown for comparison.

\section{A. Setup With Two Modulating Piezos}

The setup for the experiment, shown in Fig. 1, is partly based on previously designed instrumentation [4] that used a bulk glass reference gas cell instead of hollow-core fiber. It comprises an external cavity diode laser (ECDL), 


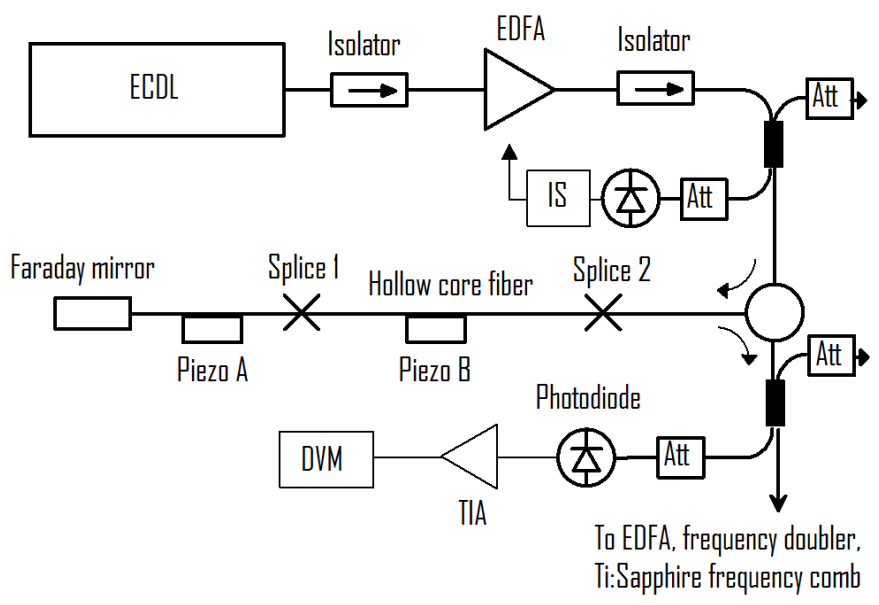

Fig. 1. Measurement setup for feasibility tests using a hollow core fiber gas cell. Att is an attenuator, IS is the intensity stabilizer, and TIA is a transimpedance amplifier.

an erbium-doped fiber amplifier (EDFA), intensity stabilization, HCF, Faraday mirror and circulator, detection, and two piezo stacks. The HCF is an approximately 40-cm long photonic crystal HCF and is spliced to telecom fiber at both ends. In addition to high reflectivity, the splices had high losses. The two piezo stacks, A and B, were glued to the telecom fiber between mirror and splice one, and to the HCF, using a cyanoacrylate glue. The outer protective layers of the telecom fiber were removed before gluing to facilitate stretching.

Piezo A and piezo B were driven by two separate, synchronized signal generators. A triangular waveform at $50 \mathrm{~Hz}$ was used for modulating the piezos. This frequency is the local mains frequency and is effectively averaged out by the DVM. Such a low frequency also resulted in good relative sharpness of the turning points of the motion of the piezos. Generally, a more robust choice would have been using a frequency different from the mains frequency with a suitable DVM time constant. However, the system was operable at similar performance level also with, e.g., a $60-\mathrm{Hz}$ piezo modulation.

By looking at the interference fringes in the detected signal with an oscilloscope, the amplitudes were tuned in such a way that each piezo oscillated over an integer number of interference fringes and the relative phase of the signals was tuned in such a way that the piezos changed direction simultaneously. Therefore, the part of the interference effects dependent on the combined stretching effect of both piezos also spread over an integer number of fringe periods. In this experiment piezo A was modulated over a few fringes and piezo B was modulated over a single fringe period. Therefore, the fringe frequencies were also different, helping to further spread the combined interference effects evenly for averaging.

The DVM was used in dc mode and averaging time was $200 \mathrm{~ms}$, effectively averaging the 50-Hz modulation. Stepping of the ECDL wavelength setting was done at 1-s intervals.

\section{B. Setup With Laser Power Modulation}

The setup with laser power modulation and frequency comb locking is shown in Fig. 2. The acetylene-filled

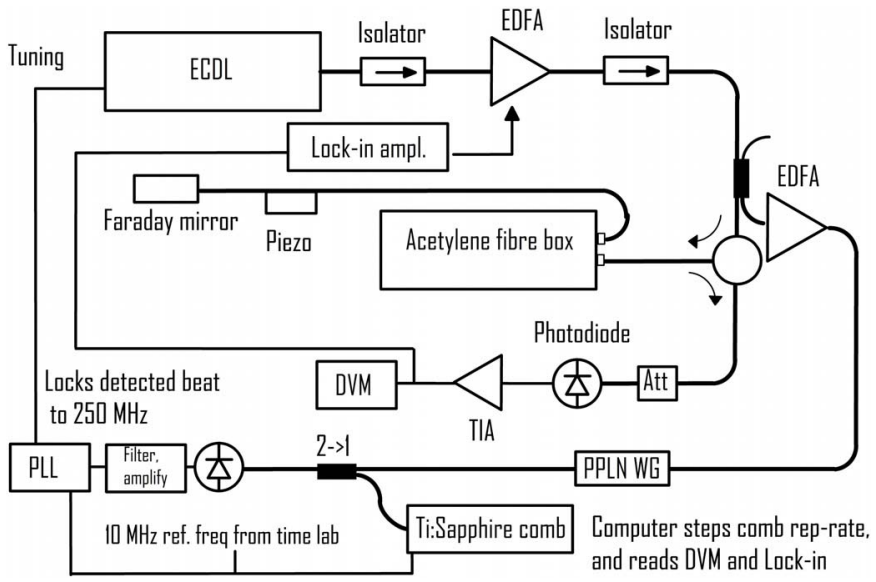

Fig. 2. Measurement setup using low-pressure acetylene-filled hollow core fiber gas cell and laser power modulation. Att is the attenuator, and TIA is the transimpedance amplifier. PLL is phase-locked loop electronics and PPLN WG is periodically poled lithium niobate waveguide.

fiber is situated inside the box denoted as acetylene fiber box. The box has Ferrule Connector/Angled Physical Contact connectors and connecting telecom fibers. The $\mathrm{HCF}$ used is a seven-cell photonic crystal fiber with a core diameter of $8 \mu \mathrm{m}$ and a length of $2.8 \mathrm{~m}$. The acetylene filling pressure has been estimated as $12 \mathrm{~Pa}$, but a pressure increase of approx. $15 \mathrm{~Pa} /$ month has been observed due to air leak. All the measurements presented here have been performed within six months of filling.

One fiber length modulating piezo was used at the fiber leading to the end mirror to reduce large (approximately 5\% amplitude compared with off-spectrum mean intensity) short-period interferences.

A lock-in amplifier (SRS type 830) was used to provide 333-Hz modulation signal to the pump laser of the first EDFA. The resulting power modulation in the EDFA output was approximately $50 \%$ of the mean power. The frequency of the lock-in amplifier was selected in such a way that the detection of the lock-in frequency would not detect much undesired signal from the piezo modulation at $50 \mathrm{~Hz}$. The averaging time constant in the lock-in amplifier was $100 \mathrm{~ms}$. The mean power entering the acetylene fiber box was approximately $10 \mathrm{~mW}$. The second EDFA was added to the system to amplify the power fed into the frequency-doubling crystal, periodically poled lithium niobate waveguide, for higher output power.

The wavelength of the ECDL was tuned close to the measured acetylene transition and then locked to the titanium-sapphire frequency comb via the frequency doubling and using phase-locked loop-type electronics [4]. The wavelength scan was done by stepping the pulse repetition rate of the frequency comb system.

\section{Setup With Two Different Wavelengths and Pump Power Modulation}

The setup with two different wavelengths for the pump and probe beam is shown Fig. 3. The light provided by the fiber laser (NKT Photonics E15) is split in a 50/50 fiber-splitter. Two acousto-optic modulators (AOMs) are used to blue-shift the light respectively $40 \mathrm{MHz}$ (probe) and 


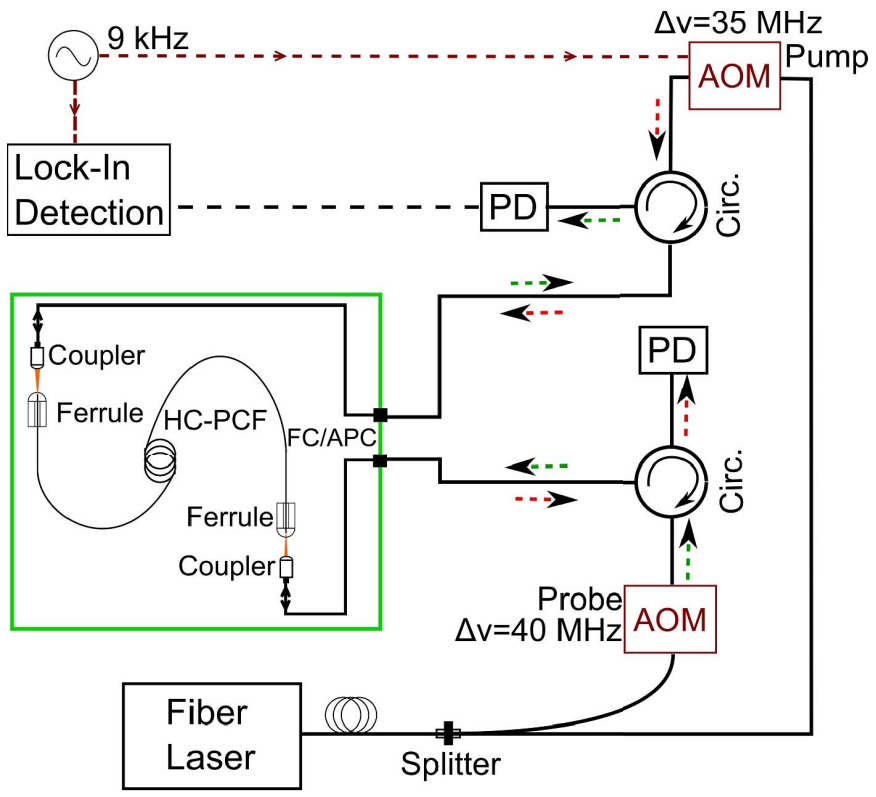

Fig. 3. Measurement setup using two different counter-propagating wavelengths $(\Delta v=5 \mathrm{MHz})$ in the hollow core fiber gas cell. The two AOMs shift the laser frequency in the same direction to maintain the sub-Doppler resonance close to the bottom of the Doppler absorption line, where the $S / N$ is higher.

$35 \mathrm{MHz}$ (pump). The pump beam is also modulated $100 \%$ in intensity by applying a $9-\mathrm{kHz}$ square wave to the pump AOM. This $9-\mathrm{kHz}$ signal is used as external reference for the lock-in amplifier (SRS830). Two circulators are used to monitor both the transmitted probe and the pump using two photodetectors (PD). The frequency is controlled by offset-locking of the laser to a second acetylene stabilized laser described in [8]. The acetylene-filled fiber is the same as the one shown in Fig. 2. The typical pump/probe power used is $5 \mathrm{~mW}$.

\section{SATURATion AND Power Modulation}

The nonlinearity of the saturation phenomenon can be exploited to differentiate true saturated absorption features from the linear absorption and interference.

For any linear loss in the setup, the ac/dc ratio remains constant, thus effectively eliminating variations arising from interference. When a gas absorber is introduced to the system, the power response becomes nonlinear and saturation can be seen through the ac/dc ratio, as can be seen from Fig. 8 . This method faithfully recovers the usual Doppler-free shape of the lamb dip on Doppler-broadened background. However, the curvature of the background is in the opposite direction, compared with the traditional transmission signal. We also note that harmonic distortion of the power modulation can be used as an indicator of nonlinear response due to saturation, but this was not investigated in this paper.

\section{RESULTS}

\section{A. Two-Piezo Modulation}

Fig. 4 shows the recorded detector signal as DVM readings over a wavelength scan with and without the

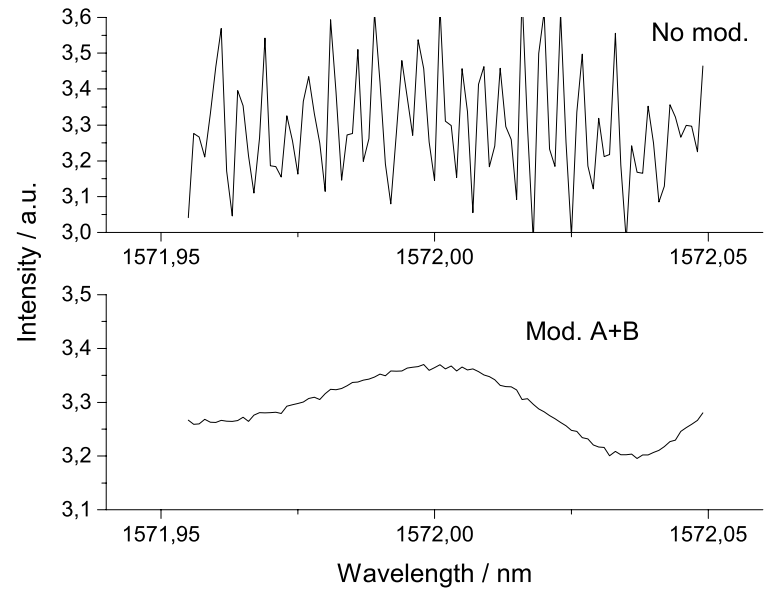

Fig. 4. Recorded intensity at detector over a wavelength scan in arbitrary units. Upper graph is without piezo modulation and lower is with both piezos on. The remaining shape is a combination of $\mathrm{CO}_{2}$ absorption spectrum and interference effects. $\mathrm{CO}_{2}$ has an absorption at $1572.02[\mathrm{R}(18)$ rotational line associated with $\left[00^{0} 0\right] \rightarrow\left[30^{0} 1\right]$ vibrational transition]. The wavelength scale is approximate in this experiment.

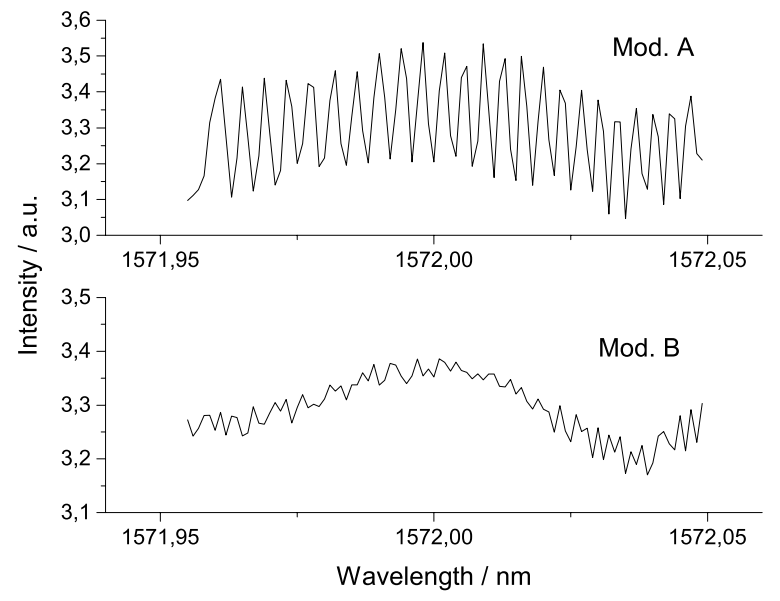

Fig. 5. Recorded intensity over a wavelength scan, with only one piezo A (upper graph) or B (lower graph) modulated.

piezo modulations. The HCF used in this experiment was filled with $\mathrm{CO}_{2}$ at atmospheric pressure.

Fig. 5 shows the corresponding scan with only piezo A or B modulated. Clearly, neither of modulations is sufficient by itself to cancel the interference out.

In the absence of any modulation, the amplitude of the interference features varied between approximately $20 \%$ and $80 \%$ of the signal mean.

\section{B. Laser Power Modulation}

Fig. 6 shows the directly measured dc-coupled detector signal as a function of laser frequency. From scan to scan, the distorted, asymmetric form varied so that the extent and direction of the slant varied. The piezo modulation of the end mirror removed short-period interferences quite effectively but there was still a distorted shape of absorption. To avoid gluing a second piezo to the HCF, an alternative method, i.e., the power modulation scheme, was applied. 


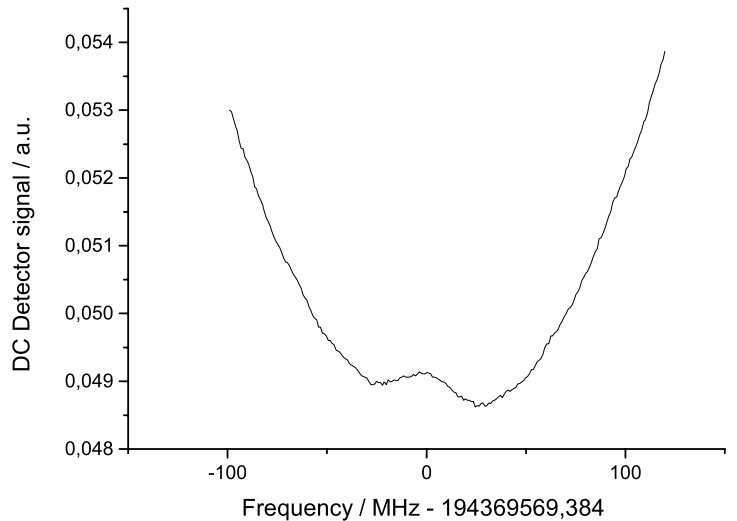

Fig. 6. Recorded dc signal in the scan over the acetylene P16 line.

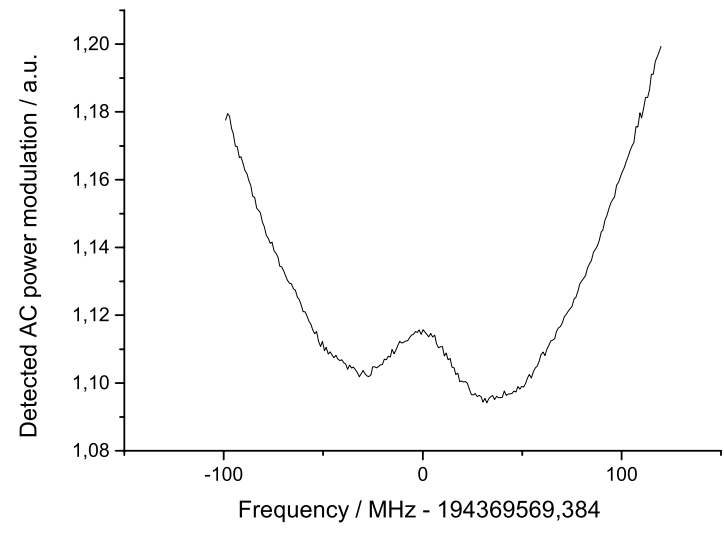

Fig. 7. Recorded ac signal in the scan over the acetylene P16 line.

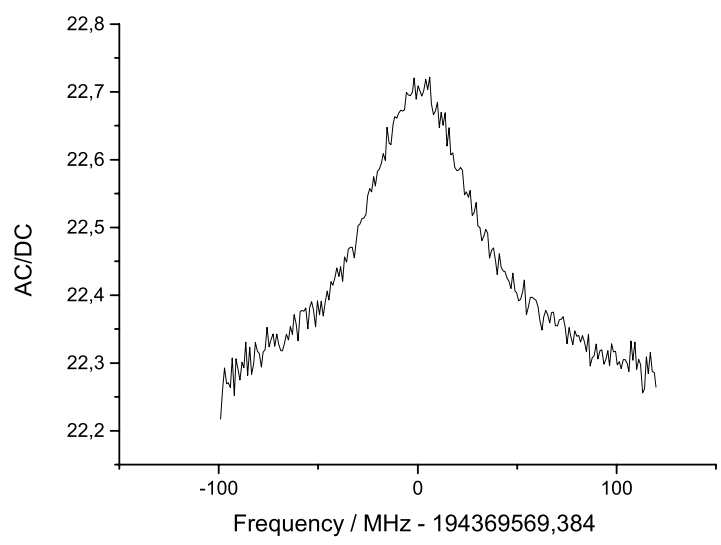

Fig. 8. AC/dc ratio in the scan over the acetylene P16 line.

The comb repetition rate was stepped at approximately 1 -s intervals, with approximately 200 measurement points in a single scan. The $1 \mathrm{~s}$ wait consisted mainly of waiting for the DVM and lock-in reading to stabilize before reading. All data in Figs. 6-9 are from a single scan.

Fig. 7 shows the ac component detected with the lock-in amplifier simultaneously with the dc component. The Lamb dip is more pronounced due to the nonlinear power response of the saturable medium, although it is still highly distorted.

Fig. 8 shows the ac signal normalized by dividing it by the dc signal. The lamb dip feature is more symmetric, protruding from the Doppler-broadened shape. The ac and dc signals are measured simultaneously from the same scan.

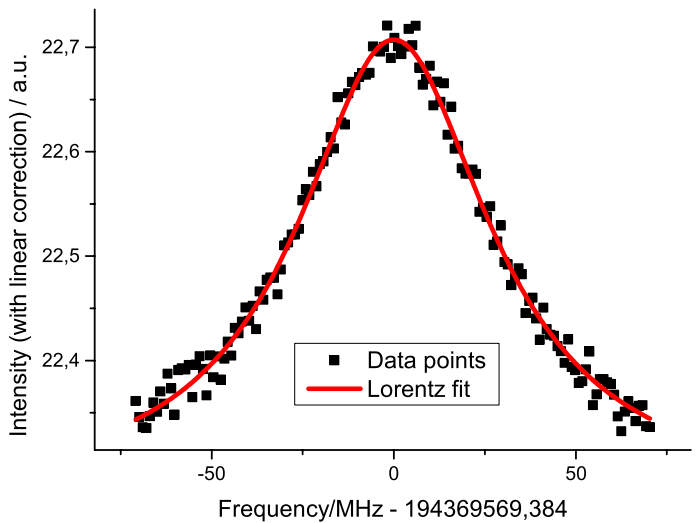

Fig. 9. AC/dc ratio in the central part of the scan over acetylene P16 line with Lorenz fit. A fitted line has been subtracted before the Lorenz fit.

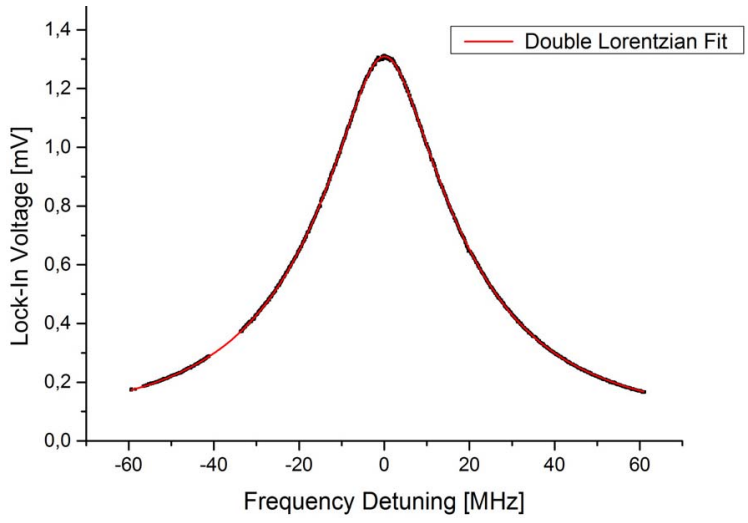

Fig. 10. Lock-in detected sub-Doppler resonance. The data are plotted shifted by $2.5 \mathrm{MHz}$, because of the different frequency shifts from the two AOMs. The missing of data at $-37.5 \mathrm{MHz}$ is due to an instability of a counter reading at a zero-crossing point.

In Fig. 9 the center part is first processed by just subtracting a fitted line to remove the small remaining tilt seen in Fig. 8. Then a Lorentzian function was fitted. The center wavelength of the fit in the figure shown differs from the reference value for low-pressure bulk glass cells only by less than $1 \mathrm{kHz}$. However, in five repeated experiments, the variation in the fitted central frequency was approximately $200 \mathrm{kHz}$, and the mean value within $100 \mathrm{KHz}$ of the bulk-cell reference value. The corresponding graphs of the other scans were similar in shape, with slightly changing noise and interference levels probably due to drifting of measurement parameters. The variation of the fitted central frequency is possibly mainly due to the limited number of data points that we were able to measure, compared with the measurement noise level. The majority of the fitted central frequencies were higher than the bulk cell reference value.

\section{Comparison With the Two Different Wavelengths Method}

Fig. 10 shows the lock-in amplifier output when demodulating the signal acquired by the PD with the external reference frequency $(9 \mathrm{kHz})$. The frequency of the laser is stepped by $100 \mathrm{KHz}$ over a $120-\mathrm{MHz}$ frequency range. Five data points are acquired per frequency step with a lock-in integration time of $100 \mathrm{~ms}$. A delay time of $100 \mathrm{~ms}$ has been set 
at the beginning of every single step due to the response time of the offset-locking setup. The total acquisition time is about $12 \mathrm{~min}$.

The data in Fig. 10 are fitted with a sum of two Lorentzian functions with individual linewidths and amplitudes but identical center frequencies. This empirical line shape is chosen to include the increased contribution from the slow molecules inside the fiber [1]. At low pressure and low optical power, slow molecules provide a narrow contribution to the signal. The faster molecules have a higher interaction rate with the core wall, destroying the coherence in the light matter interaction and broadening their contribution to the signal. The fit shows a center frequency blue-shifted by approximately $50 \mathrm{kHz}$. Eight repeated measurements show an average shift of $19 \mathrm{KHz}$ with a standard deviation of $20 \mathrm{kHz}$. The full-width at half-maximum linewidth is $(39 \pm 0.4) \mathrm{MHz}$. The pressure broadening due to the leak of the cell must be considered when compared with the measurements of Figs. 6-9. According to measurements by DFM, the linewidth should be approx. $46 \mathrm{MHz}$ at the time when tests presented in Figs. 6-9 have been performed. The leak-rate observed is under investigation to evaluate the long-term stability of the fiber-filled reference. A complete performance evaluation of the system will be presented in a future work, including a pressure stability characterization.

\section{CONCLUSiON}

The presented measurement methods provide tools for interference cancellation in HCF-based reference gas cells, and the ac/dc method could also be used to cancel interference in various other saturated absorption measurements. Detection of saturated absorption in HCF cell was shown to be possible without having the pump and probe beams at different frequency. The results suggest that repeatability in the measuring of transition center frequency is possible at least $100 \mathrm{kHz}$ level with acetylene-filled HCF cells, using different measurement techniques.

\section{REFERENCES}

[1] J. Hald, J. C. Petersen, and J. Henningsen, "Saturated optical absorption by slow molecules in hollow-core photonic band-gap fibers," Phys. Rev. Lett., vol. 98, no. 21, pp. 213902-1-213902-4, 2007.

[2] New Generation of Frequency Standards for Industry. [Online]. Available: http://projects.npl.co.uk/frequencystandards/, accessed Jan. 24, 2014.

[3] J. A. Silver and A. C. Stanton, "Optical interference fringe reduction in laser absorption experiments," Appl. Opt., vol. 27, no. 10, pp. 1914-1916, May 1988.

[4] V. Ahtee, M. Merimaa, and K. Nyholm, "Precision spectroscopy of acetylene transitions using an optical frequency synthesizer," Opt. Lett., vol. 34, no. 17, pp. 2619-2621, Sep. 2009.

[5] V. Ahtee, M. Merimaa, and K. Nyholm, "Fiber-based acetylenestabilized laser," IEEE Trans. Instrum. Meas., vol. 58, no. 4, pp. 1211-1216, Apr. 2009.

[6] J. Seppä, M. Merimaa, A. Manninen, and A. Lassila, "Interference cancellation for hollow-core fiber reference cells," in CPEM Dig., 2014, pp. 672-673.
[7] M. Merimaa, K. Nyholm, M. Vainio, and A. Lassila, "Traceability of laser frequency calibrations at MIKES," IEEE Trans. Instrum. Meas., vol. 56, no. 2, pp. 500-504, Apr. 2007.

[8] J. Hald, L. Nielsen, J. C. Petersen, P. Varming, and J. E. Pedersen, "Fiber laser optical frequency standard at $1.54 \mu \mathrm{m}$," Opt. Exp., vol. 19, no. 3, pp. 2052-2063, Jan. 2011.

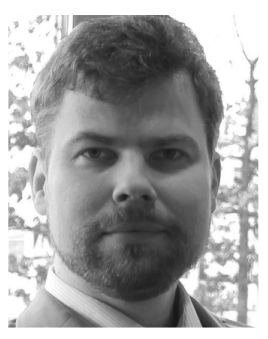

Jeremias Seppä received the M.Sc. and Ph.D. degrees from Aalto University, Espoo, Finland, in 2007 and 2015, respectively. His Ph.D. dissertation was on nanometrology and interferometry.

$\mathrm{He}$ is a Senior Scientist with the Length Metrology Group, Centre for Metrology and Accreditation, VTT Technical Research Centre of Finland, Espoo. He has been involved in computer science, mathematics, machine learning, measurement, and laser physics. His current research interests include laser frequency, nanometrology, and interferometry.

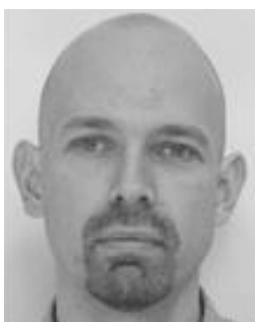

Mikko Merimaa was born in Helsinki, Finland, in 1972. He received the M.Sc. and Ph.D. degrees from Aalto University, Espoo, Finland, in 1997 and 2001, respectively. His dissertation was on frequency standards based on diode lasers.

$\mathrm{He}$ held a two-year post-doctoral position with Aalto University. He joined the Centre for Metrology and Accreditation, where he was the Leader of the Time, Frequency and Spectroscopy Group. He is currently a Research Manager with the Centre for Metrology and Accreditation, VTT Technical Research Centre of Finland, Espoo. His current research interests include time and frequency, optical clocks, and spectroscopy.

Albert Manninen, photograph and biography not available at the time of publication.

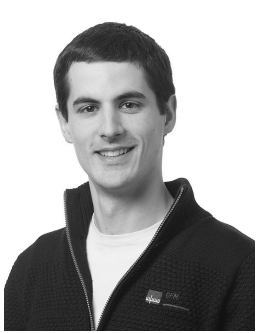

Marco Triches was born in Belluno, Italy. He received the master's degree in physics from the University of Padua, Padua, Italy, in 2012. He is currently pursuing the Ph.D. degree with DTU Fotonik, Kgs. Lyngby, Denmark.

He focused on experimental physics, taking his thesis project abroad, with DTU Nanotech, Kgs. Lyngby, Denmark, during his studies. In the group of Prof O. Hansen, he fabricated and studied the physics beyond the electret-based energy harvester, developing specific silicon micro-fabrication techniques. He currently focuses his research on frequency standard technology with Danish Metrology Institute, Kgs. Lyngby, where he is investigating the hollow core photonics crystal fiber application for laser frequency noise reduction in $1 \mathrm{~Hz}$ to $100 \mathrm{kHz}$ bandwidth.

Jan Hald, photograph and biography not available at the time of publication.

Antti Lassila, photograph and biography not available at the time of publication. 